\title{
Generalized epilepsy with febrile seizures-plus
}

INSERM

\section{Source}

INSERM. (1999). Orphanet: an online rare disease and orphan drug data base.

Generalized epilepsy with febrile seizures-plus. ORPHA:36387

Generalized epilepsy with febrile seizures plus (GEFS+) is a familial epilepsy syndrome in which family members display a seizure disorder from the GEFS+ spectrum which ranges from simple febrile seizures (FS) to the more severe phenotype of myoclonic-astatic epilepsy (MAE) or Dravet syndrome (DS) (see these terms). 